Check for updates

Cite this: J. Mater. Chem. C, 2021 9, 10811

Received 27th April 2021,

Accepted 2nd June 2021

DOI: $10.1039 / \mathrm{d} 1 \mathrm{tc0} 01943 \mathrm{k}$

rsc.li/materials-c

\title{
Plasmon-assisted spin transition in gold nanostar@spin crossover heterostructures $\dagger$
}

\author{
Roger Sanchis-Gual, (D) $\ddagger$ Ramón Torres-Cavanillas, (D) $\ddagger$ Marc Coronado-Puchau, (D) * \\ Mónica Giménez-Marqués (D) * and Eugenio Coronado (D) *
}

Herein we report the design of coreashell nanoparticles formed by a metallic Au nanostar core and a spin-crossover shell based on the coordination polymer $\left[\mathrm{Fe}(\mathrm{Htrz})_{2}(\operatorname{trz})\right]\left(\mathrm{BF}_{4}\right)$. This procedure is general and has been extended to other metallic morphologies (nanorods, nanotriangles). Thanks to the photothermal effect arising from the plasmonic properties of the Au nanostar, $60 \%$ of iron centers undergo a thermal spin transition inside the thermal hysteresis triggered by a $808 \mathrm{~nm}$ laser low intensity irradiation. Compared to other Au morphologies, the great advantage of the nanostar shape arises from the hot spots created at the branches of the nanostar. These hot spots give rise to large NIR absorptions, making them ideal nanostructures for efficiently converting light into heat using low energy light, like that provided by a $808 \mathrm{~nm}$ laser.

\section{Introduction}

The use of molecular switching materials (especially organic and metal-organic) has emerged as a very appealing possibility for their application in molecular electronics. ${ }^{1,2}$ In this context, spin-crossover materials (SCO) are one of the most promising molecular compounds. They are a particular type of coordination compound that can switch between two possible electronic configurations, namely low spin (LS) and high spin (HS), ${ }^{3}$ upon the application of an external stimulus such as temperature, pressure, light, electric field or molecular sorption in the case of porous materials. ${ }^{4-7}$ Remarkably, both electronic states display entirely different physical properties, which make them highly interesting multifunctional molecular materials. ${ }^{8-12}$ Even more, they may exhibit a strongly cooperative spin transition with a thermal hysteresis near room temperature, endowing memory to the material. ${ }^{5,13}$ This hysteresis and the transition temperatures depend on the coordinated ligands, the metal, and the compound's crystallinity. ${ }^{14}$

Among the plethora of SCO compounds, the $\left[\mathrm{Fe}(\mathrm{Htrz})_{2}(\mathrm{trz})\right]$ $\left(\mathrm{BF}_{4}\right)$ polymer ( $\mathrm{Htrz}=1,2,4$-triazole) is one of the most promising due to its high cooperativity and transition temperatures above room temperature. Indeed, these chain coordination polymers display a broad hysteresis of $40 \mathrm{~K}$, maintained even for nanoparticles (NPs) as small as $4 \mathrm{~nm} .{ }^{15-18}$

Instituto de Ciencia Molecular, Universitat de València, Catedrático José Beltran 2 , 46980, Paterna, Spain. E-mail: marc.coronado@uv.es,

monicagimenez-marques@uv.es, eugenio.coronado@uv.es

$\dagger$ Electronic supplementary information (ESI) available. See DOI: 10.1039/ d1tc01943k

\$ These authors contributed equally to this work.
Furthermore, the controlled synthesis of these compounds has permitted the design of hybrid heterostructures. ${ }^{19}$ In this context, core@shell NPs based on $\left[\mathrm{Fe}(\mathrm{Htrz})_{2}(\mathrm{trz})\right]\left(\mathrm{BF}_{4}\right)$ as core and a shell of silica or even of another SCO compound, have been successfully prepared. ${ }^{18,20-22}$ One of the most appealing hybrids combines SCO with metallic NPs to afford electronic devices based on these multifunctional nanostructures. ${ }^{23-25}$ A recent example of this electronic boosting has been reported by us in a core@shell nanostructure based on an Au core surrounded by a $\left[\mathrm{Fe}(\mathrm{Htrz})_{2}(\mathrm{trz})\right]\left(\mathrm{BF}_{4}\right)$ SCO shell. This material displayed a significant improvement in the electrical detection of the spin state thanks to the enhancement in the conductivity provided by the metallic core. ${ }^{26}$ This system was synthesized by growing the SCO shell via the alternative sequential addition of the precursors over $12 \mathrm{~nm}$ spherical $\mathrm{Au}$ in an aqueous solution and at room temperature.

Another possibility of this hybrid nanostructure is that of inducing the spin transition by low-intensity light irradiation, thanks to the heating of the nanostructure due to the plasmonic metal, which can convert light into heat. ${ }^{27}$ In this direction, $\mathrm{Au}$ NPs have been anchored to $\left[\mathrm{Fe}(\mathrm{Htrz})_{2}(\mathrm{trz})\right]\left(\mathrm{BF}_{4}\right) @ \mathrm{SiO}_{2}$ through the silica shell. ${ }^{28,29}$ For this hybrid, the power of a $633 \mathrm{~nm}$ laser irradiation required to induce the transition a few degrees before the transition temperature could be reduced by $c a .70 \% .{ }^{29}$ However, the thick $\mathrm{SiO}_{2}$ shell and the bridging molecule may reduce the heat transfer between the metal and the SCO. At the same time, the high energy of the laser may indirectly warm the sample.

Therefore, the synthesis of hybrids with metallic NPs in closer contact with the SCO compound and plasmonic nanostructures that exhibit an efficient thermal conversion by 
irradiating with lower energy laser could overcome these two problems. In this sense, an efficient light-induced spin switching has been achieved by covering lithographically patterned $\mathrm{Au}$ nanorod arrays with a molecular SCO complex film. ${ }^{30}$ In a different approach but following the same concept, a direct decoration of $\mathrm{Au}$ NPs with $\left[\mathrm{Fe}(\mathrm{Htrz})_{2}(\mathrm{trz})\right]\left(\mathrm{BF}_{4}\right)$ via the nitrogen atoms from the SCO NPs surface was explored as a manner to enhance the contact area, thus, the heat transfer. Unfortunately, no photothermal effect could be observed for this hybrid. ${ }^{31}$ Recently, this concept was improved by embedding Au nanorods in a $\left[\mathrm{Fe}(\mathrm{Htrz})_{2}(\mathrm{trz})\right]\left(\mathrm{BF}_{4}\right)$ shell. On the one hand, the plasmonicSCO interaction is enhanced due to the close contacts between both components. On the other hand, the Au nanorods photothermal conversion is maximized at lower energy lasers, thanks to the plasmon properties of the $\mathrm{Au}$ nanorods that have a maximum absorption around $830 \mathrm{~nm}$. In contrast with the direct decoration, this core@shell architecture presented a well-defined thermo-induced switching, achieving a photoconversion of $55 \%$ upon irradiation with an $830 \mathrm{~nm}$ laser inside the hysteresis. ${ }^{32}$

Herein, we present the synthesis of a core@shell structure based on an $\mathrm{Au}$ nanostar (NS) core and a $\left[\mathrm{Fe}(\mathrm{Htrz})_{2}(\operatorname{trz})\right]\left(\mathrm{BF}_{4}\right)$ shell, where the photothermal heating of the metallic core was used to induce the spin transition of the shell by low-intensity light irradiation with an $808 \mathrm{~nm}$ laser. The great advantage of the NS shape compared to other morphologies arises from the multiple hot spots created at the branches of the star (in a nanorod, for example, two hot spots are created at the two extremes of the rod). ${ }^{33}$ These hot spots give rise to prominent NIR absorptions, making them ideal for efficiently converting light into heat using low energy light like $808 \mathrm{~nm}$.

\section{Experimental section}

\subsection{Materials}

All chemical reagents were purchased and used without further purification. Chloroauric acid, 1,2,4-triazole, iron tetrafluoroborate hexahydrate, sodium citrate tribasic dihydrate, sodium borohydride hexadecyltrimethylammonium bromide (CTAB), hexadecyltrimethylammonium bromide (CTAC), L-ascorbic acid, potassium iodide and polyvinylpyrrolidone-30K (PVP) were purchased from Sigma-Aldrich. Silver nitrate was purchased from Alfa Aesar. Ultra-pure water (18.2 M $\Omega$ ) was used in the following synthesis.

\subsection{Experimental protocols}

Gold nanostar (NS). Au NPs with star-like shapes were obtained by a seeded-growth process, ${ }^{34}$ using citratestabilized Au NPs as seeds, obtained following the Turkevich method. ${ }^{35}$ For the preparation of growth solution, hydrochloric

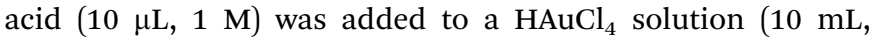
$0.25 \mathrm{mM})$. Afterward, aqueous solutions of ascorbic acid $(50 \mu \mathrm{L}$, $0.1 \mathrm{M})$ and $\mathrm{AgNO}_{3}(100 \mu \mathrm{L}, 0.003 \mathrm{M})$ were added, followed by the addition of $100 \mu \mathrm{L}$ of seed solution. Finally, PVP was added as capping agent $\left(0.5 \mathrm{~mL}, 10 \mathrm{~g} \mathrm{~L}^{-1}\right)$. After 30 minutes of incubation, the NSs suspesion was washed with water to remove the excess of PVP in the solution (Fig. S3, ESI $\dagger$ ).

NS@SCO. SCO system was grown onto PVP-stabilized NSs following a two-step approach. In a first step, a triazole aqueous solution $(250 \mu \mathrm{L}, 0.5 \mathrm{mM})$ was added into the previously prepared NSs aqueous solution (5 mL, $0.16 \mathrm{mM}$ ) under continuous stirring at $\mathrm{N}_{2}$ atmosphere for $30 \mathrm{~min}$. In a second step, iron tetrafluoroborate hexahydrate $(5 \mathrm{~mL}, 0.5 \mathrm{mM})$ and triazole ( $5 \mathrm{~mL}, 1.5 \mathrm{mM}$ ) aqueous solutions were simultaneously added $\left(4 \mathrm{~mL} \mathrm{~h}^{-1}\right)$ under stirring at $\mathrm{N}_{2}$ atmosphere and room temperature. All aqueous solutions were previously purged with Ar before starting the synthesis. The obtained core@shell NS@SCO NPs were then collected by centrifugation $(3500 \mathrm{rpm}$, $20 \mathrm{~min}$ ) and easily re-dispersed in different solvents (water, ethanol, acetone, and chloroform).

Au nanorods (NRs). These NPs were obtained through a seed-mediated growth method (Fig. S3, ESI $\dagger$ ). ${ }^{36}$

Au nanotriangles (NTs). These NPs were prepared via a seedmediated growth method (Fig. S3, ESI $\dagger$ ). ${ }^{37}$

Ag nanospheres. These NPs were synthesized via a citrate reduction protocol (Fig. S3, ESI $\dagger$ ). ${ }^{38}$

NRs@SCO, NTs@SCO and Ag@SCO. To overgrow the SCO shell a triazole aqueous solution $(200 \mu \mathrm{L}, 0.5 \mathrm{mM})$ was added to the plasmonic NP aqueous solution ( $3 \mathrm{~mL}, 0.16 \mathrm{mM})$ and the mixture was stirred for $30 \mathrm{~min}$. Then, aqueous solutions of the precursors, iron tetrafluoroborate hexahydrate $(3 \mathrm{~mL}, 0.16 \mathrm{mM})$ and triazole $(3 \mathrm{~mL}, 0.5 \mathrm{mM})$, were simultaneously added at a continuous rate $\left(4 \mathrm{~mL} \mathrm{~h}^{-1}\right)$ under stirring at room temperature. After completion of the addition, the colloidal suspension was centrifuged and washed with water.

\subsection{Characterization}

UV-vis absorption spectra were recorded on a Jasco V-670 spectrophotometer in baseline mode from 400 to $800 \mathrm{~nm}$ range, using 1.000 cm-optical-path plastic cuvettes. Transmission electron microscopy was carried out on a Technai G2 F20 microscope operating at $200 \mathrm{kV}$ and a JEOL-1010 operating at $100 \mathrm{kV}$. Samples were prepared by dropping suspensions on lacey formvar/carbon copper grids (300 mesh).

Dynamic Light Scattering (DLS) measurements were performed at room temperature with a Zetasizer Nano ZS instrument (Malvern Instruments Ltd) of the as-synthesized suspension. X-Ray Photoelectron Spectroscopy (XPS) samples were analyzed at the X-ray Spectroscopy Service at the Universidad of Valencia using a K-ALPHA Thermo Scientific spectrometer. All spectra were collected using $\mathrm{Al} \mathrm{K} \alpha$ radiation $(1486.6 \mathrm{eV})$, monochromatized by a twin crystal monochromator, yielding a focused X-ray spot (elliptical in shape with a major axis length of $200 \mu \mathrm{m}$ ) at $30 \mathrm{~mA}$ and $2 \mathrm{kV}$. The alpha hemispherical analyzer was operated in the constant energy mode with survey scan pass energies of $200 \mathrm{eV}$ to measure the whole energy band and $50 \mathrm{eV}$ in a narrow scan to selectively measure the particular elements. XPS data were analyzed with Avantage software. A smart background function was used to approximate the experimental backgrounds. Charge compensation was achieved with the system flood gun that provides low energy electrons and low energy argon ions from 
a single source. Powder X-ray diffraction (PXRD) patterns were obtained with a PANalytical X'Pert diffractometer using the copper radiation $(\mathrm{Cu}-\mathrm{K} \alpha=1.54178 \AA)$ in the $5-40^{\circ}$ region. Electrical transport measurements have been performed inside a glovebox under $\mathrm{N}_{2}$ atmosphere in the temperature range of $20-120{ }^{\circ} \mathrm{C}$, using a Keithley 4200-SCS as voltage source and electrometer. The measurements were carried out in a two-probe configuration using commercial fingerprinted electrodes, with a gap of $2.5 \mu \mathrm{m}$. The NPs were organized in the electrode gap by drop-casting a suspension of the material. Differential Scanning Calorimetry (DSC) scans were recorded in a Mettler Toledo DSC 821e model that operates in the temperature range $25-130{ }^{\circ} \mathrm{C}$ equipped with a liquid nitrogen cryostat and a $200 \mathrm{~W}$ furnace. Photocalorimetric measurements were carried out, coupling an optical fiber with an $808 \mathrm{~nm}$ laser. Attenuated total reflectance Fourier-transform infrared (ATR-FTIR) spectra were collected in an Agilent Cary 630 FTIR spectrometer in the $4000-500 \mathrm{~cm}^{-1}$.

\section{Results and discussion}

\subsection{NS@SCO preparation and characterization}

To prepare core@shell NPs employing a Au NS as metallic core and $\left[\mathrm{Fe}(\mathrm{Htrz})_{2}(\mathrm{trz})\right]\left(\mathrm{BF}_{4}\right)$ as a SCO shell, we have extended a twostep protocol developed by our group in the synthesis of $\mathrm{Au} @ S C O$ NPs formed by a spherical Au core. ${ }^{26}$ In a first step, a triazole aqueous solution $(250 \mu \mathrm{L}, 1.5 \mathrm{mM})$ is added into a previously prepared aqueous solution containing PVP stabilized NSs ( $5 \mathrm{~mL}, 0.4 \mathrm{mM}$ ) under $\mathrm{N}_{2}$ and at continuous stirring for 30 minutes, Fig. 1(i). In this step, the triazole ligand interacts with the metallic core thanks to the affinity of amines to Au. ${ }^{39}$ These triazoles act as anchoring points for the ulterior SCO growth.

In a second step, iron tetrafluoroborate hexahydrate $(5 \mathrm{~mL}$, $0.5 \mathrm{mM}$ ) and triazole $(5 \mathrm{~mL}, 1.5 \mathrm{mM})$ aqueous solutions are dropwise added $\left(4 \mathrm{~mL} \mathrm{~h}^{-1}\right)$ under a $\mathrm{N}_{2}$ atmosphere and at room temperature, Fig. 1(ii). This slow addition allows the successful Fe coordination, forming the SCO compound around the $\mathrm{Au}$ surface, while the SCO self-nucleation is minimized. The final heterostructure exhibits a total size of $102 \pm 30 \mathrm{~nm}$ with a shell size of $15 \pm 8 \mathrm{~nm}$ (Fig. 2).

Focusing on the triazole incorporation, it must be noticed that the PVP capping hinders the metallic surface obstructing the triazole anchoring. PVP is a long-chain polymer that sterically isolates the NPs, mitigating the surface reactivity and increasing the NPs colloidal stability. Therefore, despite the triazole concentration or reaction time, the PVP capping could not be replaced, as confirmed by means of ATR-FTIR and XPS; hence, the colloidal stability of the suspension over time is maintained. Thus, looking at the ATR-FTIR spectra before and after the addition of large amounts of triazole, the same bands corresponding to the $\mathrm{C}-\mathrm{N}$ vibrations at $1640 \mathrm{~cm}^{-1}$ of the PVP were clearly visible (Fig. S1, $\mathrm{ESI} \dagger$ ). XPS spectrum in the $\mathrm{N} 1 \mathrm{~s}$ region (Fig. S2, ESI $\dagger$ ) exhibits a clear peak in both spectra (i.e. the protected PVP-NS and the one mixed with the triazole), proving unequivocally the presence of PVP. From this result, we conclude that, even if the triazole is incorporated into the PVP-NS system, no substitution of the PVP capping occurs. This contrasts with the SCO growth over citrate-stabilized spherical Au NPs in which the triazole ligand partially substitutes the citrate. Notice that in order to grow the SCO shell over the NSs, the previous presence of triazole ligands is not strictly required. Still, its addition in the first step seems essential to obtain a uniform growth of the SCO shell (Fig. S7, ESI $\dagger$ ).

Finally, we observe that after the SCO growth, the colloidal stability of the resulting suspension is strongly affected. Indeed, DLS measurements indicate that a combination of single NS@SCO NPs and aggregates were present in the final solution, Fig. S12 (ESI $\dagger$ ).

The SCO formation in the hybrid was confirmed by the presence of its characteristic vibrational peaks (Fig. S1, ESI $\dagger$ ). Remarkably, the vibration signals of the PVP, located at $1640 \mathrm{~cm}^{-1}$, were still visible in the NS@SCO, indicating that the SCO wrapped the NS without replacing the PVP.

The morphology of the hybrid NS@SCO NPs was investigated through TEM, Fig. 2a. We observe that, once the addition was complete, a well-defined shell of $15 \pm 8 \mathrm{~nm}$ surrounding the metallic core is obtained, Fig. 2b and Fig. S5 (ESI $\dagger$ ). Besides, the metallic composition of the core and shell,

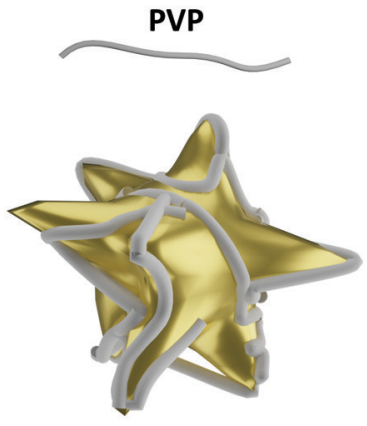

NS-PVP

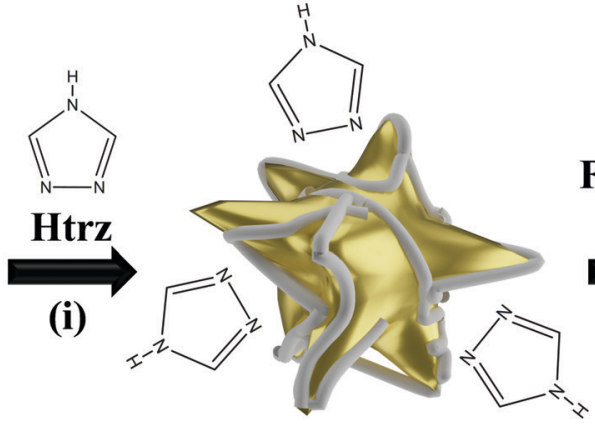

NS-Htrz

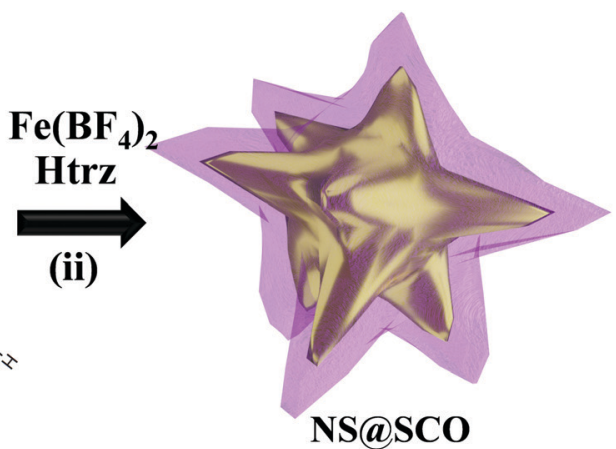

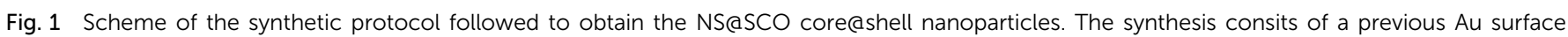
modification followed by the sequential addition of the SCO precursors. 

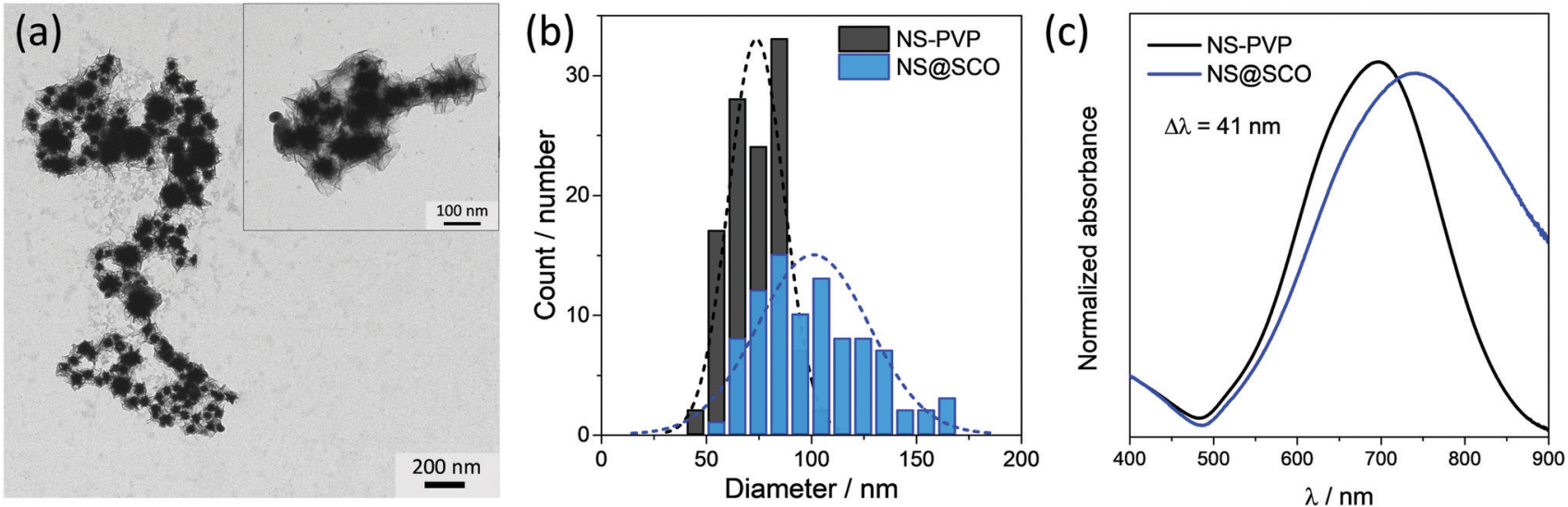

Fig. 2 (a) TEM images of the NSASCO heterostructure and (b) histograms of the size distributions for the gold core (black) and the overall coreashell nanoparticle diameter (red). These sizes have been calculated from manual counting of more than 100 NPs. (c) UV-vis spectra of the NS@SCO and the NS NPs.

examined by EDX (Fig. 3), evidences a well-defined distribution of $\mathrm{Au}$ in the core and $\mathrm{Fe}$ in the shell with a $\mathrm{Fe} / \mathrm{Au}$ ratio of 1.4.

Notice that this synthetic protocol is very versatile and reproducible and can be extended to other metallic nanostructures, as evidenced in Fig. S8-S11 (ESI $\dagger$ ) where the same approach was employed using Au nanorods, Au nanotriangles, or Ag nanospheres as the core. Nonetheless, the difficulty in obtaining some of these nanostructures and their poor colloidal stability at high concentrations have prevented us from obtaining enough core@ shell NPs to characterize them properly. In contrast, thanks to the high colloidal stability of the Au NSs provided by the PVP, large amounts of these heterostructures have been obtained, thus permitting a complete study and characterization.

Focusing on the NS@SCO heterostructure, one observes that the UV-vis spectrum is dominated by the localized plasmon band resonance (LSPR) arising from the Au (Fig. 2c). This LSPR band shifts from 697 to $738 \mathrm{~nm}$ after the SCO growth due to the change of the dielectric constant in the surrounding of the NS core during the shell formation. ${ }^{40,41}$ This indicates that, despite the PVP resilience, the SCO shell and the NS core are in intimate contact. Noticeably, this plasmon shift is considerably more pronounced than the one reported for the $\mathrm{Au}$ nanospheres covered with the same SCO compound (41 vs. $12 \mathrm{~nm}$ ). This is a consequence of the higher sensibility of the LSPR in the NSs, accounted by the Au branches. ${ }^{42}$
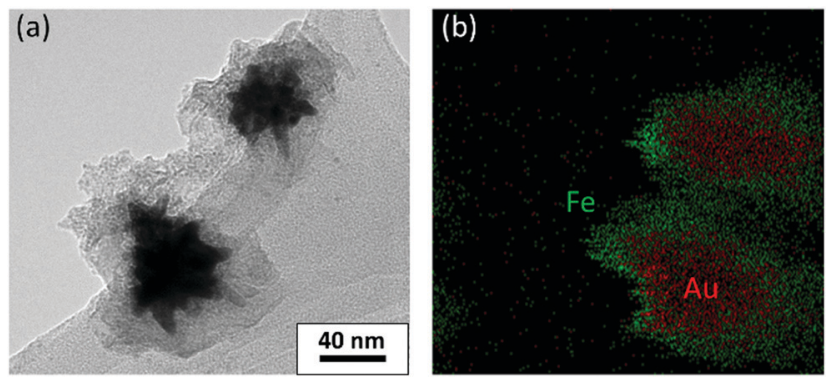

Fig. 3 (a) TEM images of the NSASCO heterostructure and (b) EDX mapping of the same area. Green and red dots correspond to Fe and $\mathrm{Au}$, respectively. The scale bar of (b) is the same as (a).
Further proof of the presence of the SCO compound in the NS@SCO heterostructure can be obtained from XPS. In this sense, the presence of iron (from the metal), nitrogen (from the ligand), and fluorine (from the counterion $\mathrm{BF}_{4}$ ) are evidenced, Fig. S2 and S6 (ESI $\dagger$ ). Taking a closer look at the Fe 2p region, a mixture of $\mathrm{Fe}^{3+}$ and $\mathrm{Fe}^{2+}$ species at the surface of the heterostructure can be discerned. On the one hand the $\mathrm{Fe}^{2+}$ bands at $708.7 \mathrm{eV}\left(\mathrm{Fe} 2 \mathrm{p}_{3 / 2}\right)$ and $721.1 \mathrm{eV}\left(\mathrm{Fe} 2 \mathrm{p}_{1 / 2}\right)$ are clearly visible, while the other bands are attributed to $\mathrm{Fe}^{3+}$. A quantification of Fe oxidation state content reveals $c a .43 \%$ of $\mathrm{Fe}^{2+}$. The high percentage of $\mathrm{Fe}^{3+}$ results from the fact that the shell is exposed and free from any surfactant moiety, being the $\mathrm{Fe}^{2+}$ susceptible to oxidize. This has been further proved by studying the XPS of the samples after $24 \mathrm{~h}$ in the air. Thus, as the Fe $2 \mathrm{p}$ region shows (Fig. S6, ESI $\dagger$ ), the SCO shell fully oxidizes in contact with ambient $\mathrm{O}_{2}$, vanishing the $\mathrm{Fe}^{2+}$ signal. It is worth mentioning that the core@shell can also be synthesized in air instead of under an inert atmosphere. However, even if the resulting NPs display spin transition, the SCO shell only contains $20 \%$ of $^{2+}{ }^{2+}$ (Fig. S6, ESI $\dagger$ ).

Finally, the crystallinity of the NS@SCO has been checked by PXRD. As it was previously reported for the Au@SCO system, no diffraction peak related to the SCO component is observed. This may come from the screening of the signal produced by the crystalline $\mathrm{Au}$, owing to its large electron density, Fig. S13 $(\mathrm{ESI} \dagger),{ }^{28}$ or to the poor crystallinity of the SCO shell due to the high mismatch between the $\left[\mathrm{Fe}(\mathrm{HTrz})_{2}(\operatorname{trz})\right]\left(\mathrm{BF}_{4}\right)$ and the $\mathrm{Au}$ crystal structures.

\subsection{Plasmon-assisted spin transition}

The spin transition of the NS@SCO was explored by calorimetric measurements (DSC), due to the high sensitivity of the technique to characterize the SCO phenomenon in such hybrids, as only the metallic centers able to switch their spin state contribute to the endo and exothermic peaks. In this technique, the typical endothermic and exothermic signals coming from the spin transition are monitored as a function of the temperature. During the first heating, a clear endothermic peak can be seen at $103{ }^{\circ} \mathrm{C}$, corresponding to the transition from LS to $\mathrm{HS}\left(T_{1 / 2}^{\mathrm{HS}}\right)$. Then, during the cooling process, the LS recovery $\left(T_{1 / 2}^{L S}\right)$ is 
evidenced by the exothermic peak located at $65{ }^{\circ} \mathrm{C}$ (blue line), Fig. 4 and Fig. S14 (ESI $\dagger$ ). These temperatures are in good agreement with the transition temperatures of $\left[\mathrm{Fe}(\mathrm{HTrz})_{2}(\mathrm{trz})\right]\left(\mathrm{BF}_{4}\right)$ NPs of comparable size, Fig. S15 and S16 (ESI $\dagger$ ). This clearly proves that the $\mathrm{Fe}^{2+}$ centers of the shell maintain the spin crossover phenomenon.

Note that after the first spin transition, the $T_{1 / 2}^{\mathrm{HS}}$ moves to $87{ }^{\circ} \mathrm{C}$ due to some irreversible changes in the crystal structure and/or evaporation of solvent molecules, whereas the transition temperature $T_{1 / 2}^{\mathrm{LS}}$ remains unaffected. ${ }^{43}$ However, despite this shift, the number of $\mathrm{Fe}^{2+}$ centers susceptible to switch their spin state seems to remain constant and without signs of fatigue after four heating and cooling cycles. This has been accounted for by the area under the curves of both peaks that remain almost constant during the successive spin transitions (Table S2, ESI $\dagger$ ).

After the DSC characterization, we explored the lightinduced spin transition by irradiating the NS@SCO sample with an $808 \mathrm{~nm}$ laser at different intensities. We chose the $808 \mathrm{~nm}$ laser because this wavelength is close to the LSPR maximum of the heterostructure (Fig. 2c), thus maximizing the light absorption and the heat released from the $\mathrm{Au}$ core. Therefore, for this experiment, we first irradiated the NS@SCO for $10 \mathrm{~min}$ with a power of $0.15 \mathrm{~mW} \mathrm{~cm}^{-2}$ at $70{ }^{\circ} \mathrm{C}$, which is a temperature located inside the thermal hysteresis. The underlying idea was to increase the local temperature around the NS core due to its photo-heating inside the hysteresis to switch the spin state of the SCO shell. We also chose that low power to reduce the laser heating in order to obtain the thermal contribution exclusively coming from the NS photo-heating. Then, the temperature was increased to $130{ }^{\circ} \mathrm{C}$, ensuring the complete transition of all the Fe centers. Finally, the sample was cooled down to $20{ }^{\circ} \mathrm{C}$ in order to switch back the SCO shell to the LS, Fig. 4.

The amount of $\mathrm{Fe}^{2+}$ centers switched to the HS during the irradiation is determined by calculating the ratio between the area under the peaks of the endo and exothermic signals, Table S2 (ESI $\dagger$ ). Interestingly, the endothermic peak area (HS transition) is 60\% lower than the exothermic (LS transition), indicating that $60 \%$ of $\mathrm{Fe}^{2+}$ centers were already switched to the HS state at $70{ }^{\circ} \mathrm{C}$ during the light irradiation. Then, we repeated this measurement at higher laser power, $0.30 \mathrm{~mW} \mathrm{~cm}{ }^{-2}$, to increase this conversion rate. Nevertheless, no significant changes were recorded. Therefore, we speculate that the $40 \%$ of not switched SCO may be located too far from the Au particle to be heated by the NS core. We decided not to measure at higher laser intensities to avoid the heating of the sample from direct irradiation.

With the aim of unequivocally attribute the transition to the photothermal effect of the NS core, two references were investigated. On the one hand, we studied the endo and exothermic areas under the curve of the NS@SCO compound following the same protocol previously described but in the absence of light to elucidate if the selected temperature $\left(70{ }^{\circ} \mathrm{C}\right.$ for 10 minutes) could induce some thermal spin transition. As shown in Table S2 (ESI $\dagger$ ) the area of the endo and exothermic signals matched perfectly in the absence of light, indicating that no spin switching occurs at this temperature. On the other hand, we also wanted to discard the light-induced spin transition as the main source of the recorded spin switching. Thus, we measured a reference sample of bare $80 \mathrm{~nm}$ NPs of $\left[\mathrm{Fe}(\mathrm{HTrz})_{2}(\mathrm{trz})\right]\left(\mathrm{BF}_{4}\right)$ analogous to the NS@SCO NPs, heating for $10 \mathrm{~min}$ at $85{ }^{\circ} \mathrm{C}$ (inside the hysteresis) and irradiating at $0.30 \mathrm{~mW} \mathrm{~cm}^{-2}$, Fig. S15 and Table S3 (ESI $\dagger$ ). Again, the exothermic peak was not affected by the light irradiation, exhibiting the same value as the endothermic peak. Thus, the laser irradiation is not optically pumping the spin state of the $\left[\mathrm{Fe}(\mathrm{HTrz})_{2}(\operatorname{trz})\right]\left(\mathrm{BF}_{4}\right)$. From these results, we conclude that $60 \%$ of the $\mathrm{Fe}^{2+}$ centers are switched as a consequence of the photothermal conversion and efficient light absorption of the plasmonic NS core.

\subsection{Electrical spin detection}

In an attempt to detect the spin transition through electrical measurements, as was previously done in spherical Au NPs surrounded by a SCO shell, ${ }^{26}$ we drop cast a NS@SCO suspension on commercial fingerprint electrodes with a gap of $2.5 \mu \mathrm{m}$. Then, temperature dependent $I-V$ curves were collected, leaving 5 minutes of thermal stabilization at each temperature. In Fig. S17 (ESI $\dagger$ ) we
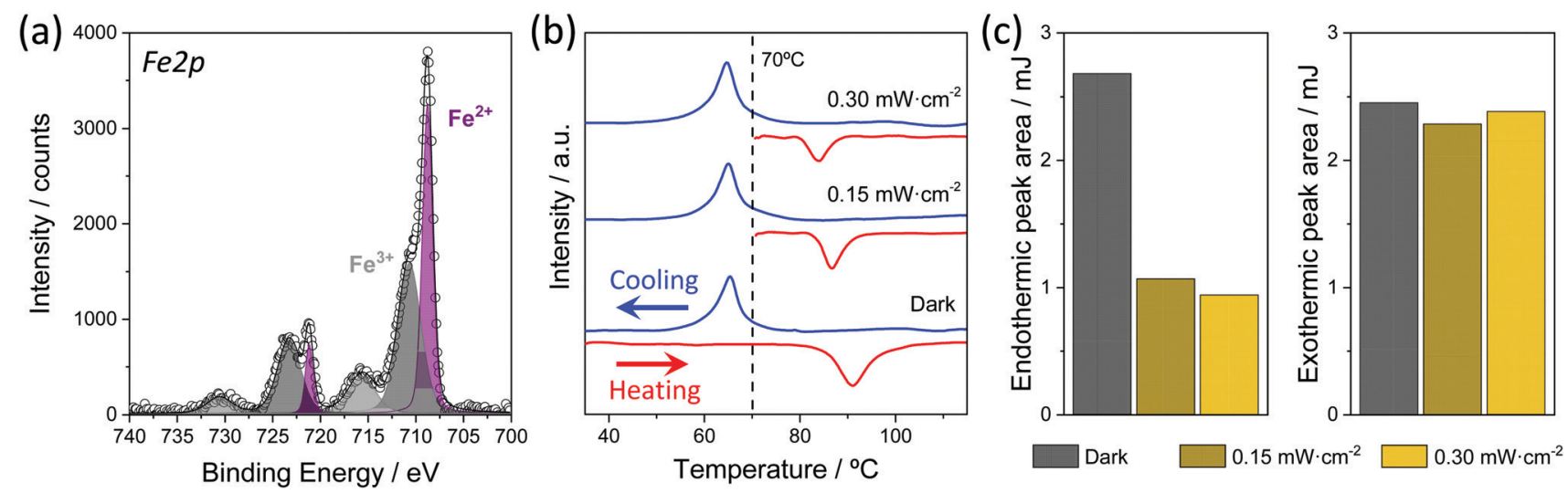

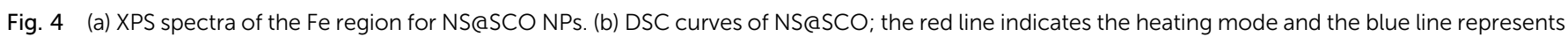
the cooling mode. (c) Variation of the endothermic and exothermic areas in different conditions. 
plot the electrical behaviour as a function of the temperature. We observe that in the range RT- $120{ }^{\circ} \mathrm{C}$, the current increases when the temperature is increased. This is the common electrical behaviour of a semiconductor or an insulator, proving that the metallic cores are not creating short-circuits thanks to the SCO insulating shell. Nevertheless, these short-circuits cannot be avoided when a complete hysteresis was attempted since, at some point, an abrupt increase in the conductance occurred in all the tested electrodes. For instance, in Fig. S17 (ESI $\dagger$ ) an example of $I-V$ curves at different temperatures are presented, showing that during the cooling, the recorded current is decreasing progressively untill $30{ }^{\circ} \mathrm{C}$, where a short-circuit occurs. This suggests that the SCO shell is being progressively damaged due to the high current density in the branches of the nanostars, heating them locally due to the Joule effect and making them collapse. In this line, we can conclude that isotropic Au NPs are more suitable in order to detect the spin transition electrically.

\section{Conclusions}

In this work, we have extended the synthetic protocol developed for $\mathrm{Au}$ nanospheres to wrap $\mathrm{Au}$ nanostars, $\mathrm{Au}$ nanorods, $\mathrm{Au}$ nanotriangles and $\mathrm{Ag}$ nanospheres with the SCO compound $\left[\mathrm{Fe}(\mathrm{Htrz})_{2}(\mathrm{trz})\right]\left(\mathrm{BF}_{4}\right)$. Here, we took advantage of the Au nanostars capabilities to efficiently convert light into heat (photothermal effect) in order to induce the spin transition of the shell by irradiating with a laser of $808 \mathrm{~nm}$ and low intensity, $0.15 \mathrm{~mW} \mathrm{~cm}^{-2}$, inside the hysteresis of the SCO (at $70{ }^{\circ} \mathrm{C}$ ). In this context, we achieved a spin switching of the $60 \%$ of SCO $\mathrm{Fe}^{2+}$ centers located in the shell. The low power guaranteed that the spin transition was induced by the photothermal effect of the metallic core. Remarkably, compared with previous works, the intensity used in this work is several orders of magnitude lower. For instance, with a $633 \mathrm{~nm}$ laser an irradiation intensity of $3000 \mathrm{~W} \mathrm{~cm} \mathrm{~cm}^{-2}$ was required to induce the complete spin transition of the $\left[\mathrm{Fe}(\mathrm{Htrz})_{2}(\mathrm{trz})\right]\left(\mathrm{BF}_{4}\right) @ \mathrm{SiO}_{2}$ system decorated with $\mathrm{Au},{ }^{29}$ whereas for $\mathrm{Au}$ nanorods embedded in SCO this intensity could be reduced to $15 \mathrm{~mW} \mathrm{~cm}^{-2}$ with a $830 \mathrm{~nm}$ laser. ${ }^{31}$ We attributed the large improvement achieved in this work mainly to two reasons: the reduced thickness of the shell, improving the heat distribution to the SCO shell, and the superior photothermal effect provided by the $\mathrm{Au}$ NSs, enhancing the light-temperature conversion. Nevertheless, other parameters related to the sample or the technique used to study the spin state may also play a role.

\section{Author contributions}

Both authors were responsible for the design, synthesis, characterization of the heterostructure, and the preparation of the manuscript. M. G.-M. and M.C.-P. were involved in the development and coordination of all experimental parts, discussion of results, and preparation of the manuscript. E. C. supervised all the work and the preparation of the manuscript. All authors revised and contributed to the presented manuscript.

\section{Conflicts of interest}

There are no conflicts to declare.

\section{Acknowledgements}

The authors acknowledge funding from the EU (ERC Advanced Grant Mol-2D 788222 and FET-OPEN COSMICS 766726), the Spanish MICINN (Unit of Excellence "Maria de Maeztu" CEX2019-000919-M and Project MAT2017-89993-R co-financed by FEDER) and the Generalitat Valenciana (Prometeo Programms PROMETEO/2017/066 and SEJI/2020/036 and Projects iDiFEDER/ 2018/061, iDiFEDER/2020/060 and iDiFEDER/2020/063; and APOSTD contract to M.C.-P. APOSTD/2019/143) and "la Caixa" Foundation (LCF/BQ/PI19/11690022). M.G.-M. thanks support from MICINN for a "Ramón y Cajal” Fellowship RYC2019-027902-I.

\section{References}

1 C. Simão, M. Mas-Torrent, N. Crivillers, V. Lloveras, J. M. Artés, P. Gorostiza, J. Veciana and C. Rovira, A Robust Molecular Platform for Non-Volatile Memory Devices with Optical and Magnetic Responses, Nat. Chem., 2011, 3(5), 359.

2 C. Simão, M. Mas-Torrent, J. Casado-Montenegro, F. Otón, J. Veciana and C. Rovira, A Three-State Surface-Confined Molecular Switch with Multiple Channel Outputs, J. Am. Chem. Soc., 2011, 133(34), 13256-13259.

3 M. A. Halcrow, Spin-Crossover Materials: Properties and Applications, 2013.

4 P. Gütlich, Y. Garcia and T. Woike, Photoswitchable Coordination Compounds, 2001, vol. 219-221.

5 J.-F. Létard, P. Guionneau and L. Goux-Capes, Spin Crossover in Transition Metal Compounds III, 2004, vol. 1, pp. 221-249.

6 C. Chemistry, Spin Crossover in Transition Metal Compounds I, 2004.

7 A. Hauser, Spin Crossover in Transition Metal Compounds II, 2004, pp. 155-198.

8 S. M. Neville, C. Etrillard, S. Asthana and J. F. Létard, LightInduced Stored Information in Nanoparticles, Eur. J. Inorg. Chem., 2010, 282-288.

9 G. Molnár, S. Rat, L. Salmon, W. Nicolazzi and A. Bousseksou, Spin Crossover Nanomaterials: From Fundamental Concepts to Devices, Adv. Mater., 2018, 30(5), 1703862.

10 C. Lefter, S. Rat, J. S. Costa, M. D. Manrique-Juárez, C. M. Quintero, L. Salmon, I. Séguy, T. Leichle, L. Nicu, P. Demont, A. Rotaru, G. Molnár and A. Bousseksou, Current Switching Coupled to Molecular Spin-States in LargeArea Junctions, Adv. Mater., 2016, 28(34), 7508-7514.

11 M. D. Manrique-Juárez, F. Mathieu, A. Laborde, S. Rat, V. Shalabaeva, P. Demont, O. Thomas, L. Salmon, T. Leichle, L. Nicu, G. Molnár and A. Bousseksou, Micromachining-Compatible, Facile Fabrication of Polymer Nanocomposite Spin Crossover Actuators, Adv. Funct. Mater., 2018, 28(29), 1801970.

12 J. Dugay, M. Giménez-Marqués, W. J. Venstra, R. TorresCavanillas, U. N. Sheombarsing, N. Manca, E. Coronado and 
H. S. J. Van Der Zant, Sensing of the Molecular Spin in SpinCrossover Nanoparticles with Micromechanical Resonators, J. Phys. Chem. C, 2019, 123(11), 6778-6786.

13 O. Kahn, Jay Martinez and C. Spin-Transition, Polymers: From Molecular Materials Toward Memory Devices, Science, 1998, 279(5347), 44-48.

14 A. Hauser, Ligand Field Theoretical Considerations, 2004, pp. 49-58.

15 E. Coronado, J. R. Galán-Mascarós, M. Monrabal-Capilla, J. García-Martínez and P. Pardo-Ibáñez, Bistable SpinCrossover Nanoparticles Showing Magnetic Thermal Hysteresis near Room Temperature, Adv. Mater., 2007, 19(10), 1359-1361.

16 J. R. Galán-Mascarós, E. Coronado, A. Forment-Aliaga, M. Monrabal-Capilla, E. Pinilla-Cienfuegos and M. Ceolin, Tuning Size and Thermal Hysteresis in Bistable Spin Crossover Nanoparticles, Inorg. Chem., 2010, 49(12), 5706-5714.

17 M. Giménez-Marqués, García-Sanz de Larrea, M. L. Coronado and E. Unravelling, the Chemical Design of Spin-Crossover Nanoparticles Based on Iron(Ii)-Triazole Coordination Polymers: Towards a Control of the Spin Transition, J. Mater. Chem. C, 2015, 3(30), 7946-7953.

18 R. Torres-Cavanillas, L. Lima-Moya, F. D. Tichelaar, H. W. Zandbergen, M. Giménez-Marqués and E. Coronado, Downsizing of Robust Fe-Triazole@ $\mathrm{SiO}_{2}$ Spin-Crossover Nanoparticles with Ultrathin Shells, Dalton Trans., 2019, 48, 15465-15469.

19 C. M. Quintero, G. Félix, I. Suleimanov, J. S. Costa, G. Molnár, L. Salmon, W. Nicolazzi and A. Bousseksou, Hybrid Spin-Crossover Nanostructures, Beilstein J. Nanotechnol., 2014, 5(1), 2230-2239.

20 S. Titos-Padilla, J. M. Herrera, X. W. Chen, J. J. Delgado and E. Colacio, Bifunctional Hybrid $\mathrm{SiO}_{2}$ Nanoparticles Showing Synergy between Core Spin Crossover and Shell Luminescence Properties, Angew. Chem., Int. Ed., 2011, 50(14), 3290-3293.

21 J. M. Herrera, S. Titos-Padilla, S. J. A. Pope, I. Berlanga, F. Zamora, J. J. Delgado, K. V. Kamenev, X. Wang, A. Prescimone, E. K. Brechin and E. Colacio, Studies on Bifunctional Fe(II)-Triazole Spin Crossover Nanoparticles: Time-Dependent Luminescence, Surface Grafting and the Effect of a Silica Shell and Hydrostatic Pressure on the Magnetic Properties, J. Mater. Chem. C, 2015, 3(30), 7819-7829.

22 Y. X. Wang, D. Qiu, S. F. Xi, Z. D. Ding, Z. Li, Y. Li, X. Ren and Z. G. Gu, Iron(II)-Triazole Core-Shell Nanocomposites: Toward Multistep Spin Crossover Materials, Chem. Commun., 2016, 52(51), 8034-8037.

23 A. Rotaru, I. A. Gural'skiy, G. Molnár, L. Salmon, P. Demont and A. Bousseksou, Spin State Dependence of Electrical Conductivity of Spin Crossover Materials, Chem. Commun., 2012, 48(35), 4163-4165.

24 A. Holovchenko, J. Dugay, M. Giménez-Marqués, R. TorresCavanillas, E. Coronado and H. S. J. van der Zant, Near Room-Temperature Memory Devices Based on Hybrid Spin-

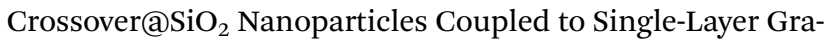
phene Nanoelectrodes, Adv. Mater., 2016, 28(33), 7228-7233.
25 J. Dugay, W. Evers, R. Torres-Cavanillas, M. GiménezMarqués, E. Coronado and H. S. J. Van Der Zant, Charge Mobility and Dynamics in Spin-Crossover Nanoparticles Studied by Time-Resolved Microwave Conductivity, J. Phys. Chem. Lett., 2018, 9(19), 5672-5678.

26 R. Torres-Cavanillas, R. Sanchis-Gual, J. Dugay, M. CoronadoPuchau, M. Giménez-Marqués and E. Coronado, Design of Bistable Gold@Spin-Crossover Core-Shell Nanoparticles Showing Large Electrical Responses for the Spin Switching, Adv. Mater., 2019, 31(27), 1900039.

27 G. Baffou and R. Quidant, Thermo-Plasmonics: Using Metallic Nanostructures as Nano-Sources of Heat, Laser Photonics Rev., 2013, 7(2), 171-187.

28 D. Qiu, L. Gu, X. L. Sun, D. H. Ren, Z. G. Gu and Z. Li, SCO@ $\mathrm{SiO}_{2} @ A u$ Core-Shell Nanomaterials: Enhanced PhotoThermal Plasmonic Effect and Spin-Crossover Properties, RSC Adv., 2014, 4(106), 61313-61319.

29 I. Suleimanov, J. Sánchez Costa, G. Molnár, L. Salmon and A. Bousseksou, The Photo-Thermal Plasmonic Effect in Spin Crossover@silica-Gold Nanocomposites, Chem. Commun., 2014, 50(86), 13015-13018.

30 K. Abdul-Kader, M. Lopes, C. Bartual-Murgui, O. Kraieva, E. M. Hernández, L. Salmon, W. Nicolazzi, F. Carcenac, C. Thibault, G. Molnár and A. Bousseksou, Synergistic Switching of Plasmonic Resonances and Molecular Spin States, Nanoscale, 2013, 5(12), 5288-5293.

31 F. Guillaume, Y. A. Tobon, S. Bonhommeau, J. F. Létard, L. Moulet and E. Freysz, Photoswitching of the Spin Crossover Polymeric Material $\left[\mathrm{Fe}(\mathrm{Htrz})_{2}(\mathrm{Trz})\right]\left(\mathrm{BF}_{4}\right)$ under Continuous Laser Irradiation in a Raman Scattering Experiment, Chem. Phys. Lett., 2014, 604, 105-109.

32 M. Palluel, N. M. Tran, N. Daro, S. Buffière, S. Mornet, E. Freysz and G. Chastanet, The Interplay between Surface Plasmon Resonance and Switching Properties in Gold@ Spin Crossover Nanocomposites, Adv. Funct. Mater., 2020, 30, 2000447.

33 L. Rodriguez-Lorenzo, R. A. Alvarez-Puebla, F. J. G. de Abajo and L. M. Liz-Marzán, Surface Enhanced Raman Scattering Using Star-Shaped Gold Colloidal Nanoparticles, J. Phys. Chem. C, 2010, 114(16), 7336-7340.

34 Y. Wang, A. B. Serrano, K. Sentosun, S. Bals and L. M. LizMarzán, Stabilization and Encapsulation of Gold Nanostars Mediated by Dithiols, Small, 2015, 11(34), 4314-4320.

35 J. Turkevich, Colloidal Gold. Part I, Gold Bull., 1985, 18(4), 125-131.

36 B. Nikoobakht and M. A. El-Sayed, Preparation and Growth Mechanism of Gold Nanorods (NRs) Using SeedMediated Growth Method, Chem. Mater., 2003, 15(10), 1957-1962.

37 L. Scarabelli, M. Coronado-Puchau, J. J. Giner-Casares, J. Langer and L. M. Liz-Marzán, Monodisperse Gold Nanotriangles: Size Control, Large-Scale Self-Assembly, and Performance in Surface-Enhanced Raman Scattering, ACS Nano, 2014, 8(6), 5833-5842.

38 Y. Wan, Z. Guo, X. Jiang, K. Fang, X. Lu, Y. Zhang and N. Gu, Quasi-Spherical Silver Nanoparticles: Aqueous Synthesis 
and Size Control by the Seed-Mediated Lee-Meisel Method, J. Colloid Interface Sci., 2013, 394(1), 263-268.

39 C. Subramaniam, R. T. Tom and T. Pradeep, On the Formation of Protected Gold Nanoparticles from AuCl 4By the Reduction Using Aromatic Amines, J. Nanopart. Res., 2005, 7(2-3), 209-217.

40 M. M. Miller and A. A. Lazarides, Sensitivity of Metal Nanoparticle Surface Plasmon Resonance to the Dielectric Environment, J. Phys. Chem. B, 2005, 109(46), 21556-21565.
41 K. M. Mayer and J. H. Hafner, Localized Surface Plasmon Resonance Sensors, Chem. Rev., 2011, 111(6), 3828-3857.

42 A. Shiohara, J. Langer, L. Polavarapu and L. M. Liz-Marzán, Solution Processed Polydimethylsiloxane/Gold Nanostar Flexible Substrates for Plasmonic Sensing, Nanoscale, 2014, 6(16), 9817-9823.

43 S. Brooker, Spin Crossover with Thermal Hysteresis: Practicalities and Lessons Learnt, Chem. Soc. Rev., 2015, 44(10), 2880-2892. 